\title{
Ansiedad, depresión y trastorno de la imagen corporal en pacientes que consultan en la Unidad de Obesidad del Hospital de Clínicas
}

\author{
Claudia Analía Parodi \\ Cátedra y Servicio de Psiquiatría-Facultad de Ciencias Médicas de la Universidad Nacional de Asunción, \\ Paraguay
}

Cómo referenciar este artículo/ How to reference this article:
Parodi CA. Ansiedad, Depresión e Imagen Corporal en pacientes que consultan en la Unidad de Obesidad del Hospital de Clínicas. Mem. Inst. Investig. Cienc. Salud. 2015;13(3):64-74

\section{R E S U M E N}

La obesidad es una epidemia mundial y constituye una enfermedad de etiología multifactorial. Debido a esta característica, su tratamiento debe ser también multidisciplinario. Existen pocos datos en la literatura acerca de las características emocionales y cognitivas, conductuales y psicopatológicas de los pacientes con obesidad en la edad adulta, sobre todo en nuestra región. El objetivo de este estudio fue identificar la presencia de psicopatología en pacientes que consultan en la Unidad de Obesidad del Hospital de Clínicas. Este estudio observacional, descriptivo, de corte transverso incluyó en forma consecutiva a 69 pacientes que consultaron en la Unidad de Obesidad del Hospital de Clínicas de octubre a noviembre de 2014. El muestreo fue de tipo no probabilístico, de casos consecutivo. El diagnóstico de sobrepeso y obesidad se realizó de acuerdo a la escala de Índice de Masa Corporal (IMC). Las entidades psicopatológicas investigadas fueron ansiedad y depresión mediante la versión española de la HADS (Hospital Anxiety and Depression Scale) que fue autoadministrada. La existencia de trastorno de la imagen corporal también fue evaluada mediante un instrumento autoadministrado. El $88,4 \%$ de los pacientes fue de sexo femenino y la edad media 42,8 años (DE: 12,7 ). El $43 \%$ de los pacientes presentó obesidad grado III. La prevalencia de ansiedad fue $18,8 \%$ y de depresión $8,7 \%$. El $44,9 \%$ de los pacientes presentaron trastorno de la imagen corporal. Los resultados coinciden con los datos encontrados en literatura internacional, encontrando una prevalencia aumentada de psicopatología en pacientes que consultan por Obesidad. Se registraron datos con respecto a descontrol alimentario, lo que indica la necesidad de aplicar herramientas validadas para el diagnóstico de Trastorno por Atracones

Palabras clave: obesidad, ansiedad, depresión, imagen corporal, psicopatología.

\section{Anxiety, depression and body image disorder in patients attending the Obesity Unit of the Hospital de Clínicas}

\section{A B S T R A C T}

Obesity is a worldwide epidemic disease and it has multifactorial etiology. Due to this, it requires a multidisciplinary treatment. There are few data about the emotional, cognitive, behavioral and psychopathological features of adult obese patients especially in our region. The objective was to identify the presence of psychopathology in patients consulting at the Obesity Unit of the Hospital de Clinicas. The diagnosis of overweight and obesity was done according to the scale of Body Mass Index (BMI). Anxiety and depression were the psychopathological entities investigated using the Spanish version of the Hospital Anxiety and Depression Scale (HADS) that was self-administered. The presence of body image disorder was also evaluated through a self-administered instrument. Of all patients, $88.4 \%$ was female and the mean age was $42.8 \pm 12.7$ years old and $43 \%$ of patients belonged to Fecha de recepción: febrero 2015. Fecha de aceptación: junio 2015

Autor correspondiente: Claudia Parodi Cátedra y Servicio de Psiquiatría-Facultad de Ciencias Médicas de la Universidad Nacional de Asunción, Paraguay 
class III obesity. Anxiety had a prevalence of $18.8 \%$, and depression $8.7 \%$. . Body image disorders were present in $44.9 \%$ of all patients. These results agree with the data found in the international literature. There was an increased prevalence of psychopathology in patients consulting for obesity. Uncontrolled eating habits were also recorded suggesting the need for the application of validated instruments for Binge Eating Disorder.

Keywords: obesity, anxiety, depression, body image, psychopathology.

\section{INTRODUCCIÓN}

La obesidad es considerada hoy en día una epidemia global. Afecta a gran parte de la población adulta e incluso está aumentado a pasos agigantados entre la población infantil y adolescente. Estudios describen un aumento de la prevalencia de obesidad desde la década del 60 de $13 \%$ a $36 \%$ durante 2009-2010 en población adulta. Por otro lado, en la población infantil el aumento fue de 5 a $17 \%$ (1). La obesidad es una de las principales causas de muerte prevenibles a nivel mundial. Según la OMS, el sobrepeso y la obesidad son el sexto factor principal de riesgo de defunción en el mundo. Cada año fallecen alrededor de 3,4 millones de personas adultas como consecuencia del sobrepeso o la obesidad. Además, el $44 \%$ de la carga de diabetes, el $23 \%$ de la carga de cardiopatías isquémicas y entre el $7 \%$ y el $41 \%$ de la carga de algunos cánceres son atribuibles al sobrepeso y la obesidad (2).

La alimentación es una necesidad básica que está relacionada no solo con aspectos biológicos, si no también psicológicos y socioculturales. La alimentación depende de circuitos neuronales primarios para su modulación. Al mismo tiempo, el aparato psíquico ejerce mecanismos de control y regulación de la ingesta a través de circuitos emocionales. Además, el acto de comer no solo implica relacionarse con los alimentos, sino que también implica rituales y relaciones con los otros y con la cultura propia (5). Si bien la obesidad en sí misma no está considerada por el Manual DSM V ni el CIE 10 $(3,4)$ como una patología psiquiátrica, si se ha reconocido el vínculo entre procesos emocionales e ingesta alimentaria

Cuando el acto de comer se desvía de sus funciones originales, para atender a alguna necesidad emocional, el comer se vuelve un acto psíquico que podría estar dirigido a elaborar la realidad, obtener placer o relacionarse con otros en relaciones conflictivas (5).

El hambre emocional se refiere a la utilización de la comida como una estrategia de afrontamiento utilizada para lidiar con emociones negativas $(6,7)$. El Trastorno por Atracón es un trastorno alimentario caracterizado por la presencia de atracones sin conductas compensatorias y que se acompaña por sensación de pérdida de control al comer (4). La prevalencia del Trastorno por Atracón en la población general es de 1,9 a $3,5 \%(8)$, sin embargo aumenta a 20 a $30 \%$ en pacientes obesos $(5,9)$.

El Trastorno por Atracón no es la única patología psiquiátrica que ha sido asociada con la obesidad. La presencia de patologías psiquiátricas en pacientes obesos ha sido estudiada, encontrándose que entre el 40 y $72 \%$ (11) de los pacientes presentan al menos una patología psiquiátrica, siendo las más frecuentes la Depresión y la Ansiedad $(11,12)$. Se ha encontrado asociación entre Depresión y obesidad, sobre todo al comparar la sintomatología depresiva con el Indice de Masa Corporal (IMC), observándose que a mayor IMC se observa puntuación más alta en escalas de Depresión (7). Faith et al. en un análisis prospectivo de asociaciones obesidadDepresión y Depresión-obesidad en población general, encontraron que la obesidad incrementa el riesgo de desarrollar Depresión (8). Sin embargo, la asociación Depresión-obesidad ha mostrado resultados contradictorios en distintos estudios $(6,8)$.

Además de la asociación de patologías psiquiátricas con obesidad, es también relevante la asociación entre alimentación y la construcción de la imagen corporal. La construcción de la imagen corporal es un proceso que se relaciona con los valores estéticos promovidos por cada sociedad en el momento que se vive. Actualmente el ideal estético asocia belleza con delgadez, tamaño que en algunos casos es difícil de alcanzar. La insatisfacción corporal se define como el malestar subjetivo de una persona con respecto a su tamaño o silueta (14) y se da principalmente cuando el tamaño corporal real difiere del ideal propuesto por los cánones sociales (15). La insatisfacción corporal se ha descrito como un factor que contribuye al malestar emocional y que 
incluso podría colaborar con el desarrollo de patologías psiquiátricas como la depresión e incluso trastornos alimentarios, especialmente si se inicia a edades tempranas (14).

Existen múltiples instrumentos utilizables para medir objetivamente la presencia de psicopatología en diferentes poblaciones de pacientes. Una de ellas es la escala HADS (Hospital Anxiety and Depresion Scale). La escala HAD es una escala breve, autoaplicada, que consta de dos subescalas: ansiedad y depresión que fue desarrollada por Zigmond y Snaith para detectar casos de ansiedad y depresión en población hospitalaria no psiquiátrica (13).

Clásicamente las conductas aberrantes relacionadas con la alimentación, sus relaciones con la psicopatología y las actitudes hacia el cuerpo y la silueta se han estudiado y descrito en población pediátrica (niños y adolescentes) y en mujeres jóvenes por constituir estos los grupos de riesgo para el desarrollo de Trastornos Alimentarios con sus principales consecuencias físicas: la obesidad y el bajo peso. Pocos estudios a nivel mundial recogen datos específicos sobre población adulta y sus relaciones con la alimentación y el cuerpo $(14,15)$.

A nivel regional existen solo algunos estudios pequeños que recogen ciertas características emocionales de la población obesa y su asociación con la psicopatología. No existen estudios realizados a nivel país sobre este aspecto de la obesidad. El objetivo de este trabajo fue realizar una aproximación al perfil emocional de los pacientes obesos en nuestro medio, que además de ofrecer una visión general de la situación actual, busca servir de base para el posterior diseño de intervenciones más eficaces en el tratamiento de la obesidad desde la perspectiva de la salud mental.

\section{PACIENTES Y MÉTODO}

Se realizó un estudio observacional, descriptivo, de corte transverso que incluyó pacientes que consultaron en la Unidad de Obesidad del Hospital de Clínicas de octubre a noviembre de 2014. El muestreo fue de tipo no probabilístico, de casos consecutivo.

Datos demográficos: Se tomaron datos sobre sexo y edad de los pacientes. En cuanto a la edad, solo se incluyeron pacientes mayores de 18 años. Otros datos considerados fueron el estado civil (en pareja, soltero, viudo) y la escolaridad (analfabeto, primaria, secundaria, terciaria).

Datos antropométricos y otros datos clínicos. El diagnóstico de obesidad se realizó utilizando el IMC que se calculó dividiendo el peso por la altura al cuadrado $(\mathrm{kg} / \mathrm{m} 2)$. Se aplicó la clasificación de IMC para adultos de la OMS.

Se incluyeron datos sobre el tiempo de obesidad a la fecha de la primera consulta, la existencia de tentativas dietéticas previas a la consulta actual. Además, se utilizó el reporte de hábitos y conductas alimentarias de cada paciente, recogidos en la ficha de evaluación nutricional de cada paciente.

Ansiedad y Depresión: Los síntomas de ansiedad y depresión fueron evaluados con la HADS (Hospital Anxiety and Depresion Scale), escala autoaplicada, que completaron los pacientes que acudieron a consultar a la Unidad de Obesidad en cualquiera de sus especialidades. Dicha escala consta de 14 ítems, 7 de los cuales corresponden a la subescala depresión y 7 a la subescala de ansiedad. Los puntuaciones entre 0 y 7 corresponden a la ausencia de trastornos, entre 8 y 11 puntos indican probabilidad de patología, y las puntuaciones mayores a 11 indican diagnóstico presente. En los casos de pacientes con problemas para completar la escala (problemas de visión, problemas de lecto escritura) la misma fue administrada por un médico especialista en psiquiatría. Trastorno de la imagen corporal: Las actitudes y creencias con respecto al peso y silueta corporal fueron medidas utilizando un cuestionario de imagen corporal que consta de 12 ítems que evalúan las actitudes y pensamientos de los pacientes dirigidos hacia su cuerpo. Estos ítems son evaluados en su intensidad recibiendo un puntaje que va de 0 (nunca) a 3 (siempre)

Para determinar indicadores de trastorno de la imagen corporal se administró un cuestionario de imagen corporal también completado por los propios pacientes en la sala de espera de la unidad. El resto de las variables se obtuvieron de los datos que constan en las fichas estandarizadas de los pacientes de la Unidad de Obesidad.

Todos los pacientes recibieron una hoja de consentimiento informado para la participación en el estudio, donde se explicó claramente el objetivo del estudio, además que su participación era voluntaria y podían rehusarse si así lo consideraban, lo cual no tendría influencia en el tratamiento que estuviera recibiendo. Se mantuvo en todo 
momento la confidencialidad de los datos recolectados. Se ofreció consejo y tratamiento a aquellos pacientes que lo requirieron o solicitaron.

Tamaño de muestra. Para estimar el tamaño de muestra se tuvo en cuenta una proporción esperada de trastornos de Ansiedad de 38\% y de Trastornos Depresivos de $8 \%$. Tomando una amplitud de $28 \%$, el tamaño mínimo de pacientes a reclutar fue 42 .

Análisis de datos. Los resultados fueron presentados en tablas y gráficos como frecuencia absoluta y relativa porcentual.

\section{RESULTADOS}

Fueron incluidos en el estudio 69 pacientes entre 18 y 78 años de edad (edad media \pm desvío estándar: $42,8 \pm 12,7$ años). El $88,4 \%$ era del sexo femenino y $56,5 \%$ tenía escolaridad terciaria (Tabla 1 ).

Tabla 1. Características demográficas de los pacientes. $n=69$

\begin{tabular}{lll}
\hline Características demográficas & Frecuencia & Porcentaje \\
\hline Sexo & & \\
Femenino & 61 & 88,4 \\
Masculino & 8 & 11,6 \\
Edad (media \pm DE) años & $\mathbf{4 2 , 8 \pm 1 2 , 7}$ & Rango: 18-78 \\
$<30$ & 11 & 15,9 \\
30 a 39 & 18 & 26,1 \\
40 a 49 & 20 & 29,0 \\
50 a 59 & 14 & 20,3 \\
$>60$ & 6 & 8,7 \\
Escolaridad & & \\
Primaria & 6 & 8,7 \\
Secundaria & 24 & 34,8 \\
Terciaria & 39 & 56,5 \\
\hline
\end{tabular}

Mayor proporción (43\%) de los pacientes presentaba obesidad grado III, encontrándose la mayor frecuencia en el grupo etario de 40 a 49 años (Tabla 2).

Tabla 2. Distribución del Índice de Masa Corporal por edad en pacientes que consultan en la Unidad de Obesidad del Hospital de Clínicas. $n=69$

\begin{tabular}{llllll}
\hline Franja etaria & $\begin{array}{l}\text { Obesidad } \\
\text { Sobrepeso }\end{array}$ & Grado 1 & Grado 2 & Grado 3 & Total \\
\hline$<30$ & $1(9,1 \%)$ & $1(9,1 \%)$ & $5(45,5 \%)$ & $4(36,4 \%)$ & 11 \\
30 a 39 & - & $4(22,2 \%)$ & $6(33,3 \%)$ & $8(44,4 \%)$ & 18 \\
40 a 49 & $2(10,0 \%)$ & $3(15,0 \%)$ & $3(15,0 \%)$ & $12(60,0 \%)$ & 20 \\
50 a 59 & - & - & $6(42,9 \%)$ & $8(57,1 \%)$ & 14 \\
$\geq 60$ & - & $2(33,3 \%)$ & $3(50,0 \%)$ & $1(16,7 \%)$ & 6 \\
Total & $3(4,3 \%)$ & $10(14,5 \%)$ & $23(33,3 \%)$ & $33(43 \%)$ & 69
\end{tabular}

El $36,2 \%$ de los pacientes tenía entre 10 y 20 años de obesidad a la fecha de la primera consulta. El 82,6\% había realizado varios intentos de descenso de peso (Tabla 3). 
Tabla 3. Tiempo de obesidad y tentativas dietéticas previas a la consulta en pacientes que consultan en la Unidad de Obesidad del Hospital de Clínicas. $n=69$

\begin{tabular}{lll}
\hline & Frecuencia & Porcentaje \\
\hline Tiempo de obesidad & & \\
$<10$ & 20 & 29,0 \\
$10-20$ & 25 & 36,2 \\
$>20$ & 24 & 34,8 \\
Tentativas dietéticas & & \\
0 & 4 & 5,8 \\
1 & 8 & 11,6 \\
$>1$ & 57 & 82,6 \\
\hline
\end{tabular}

\section{Escala HAD}

El trastorno de ansiedad se presentó en el $18,8 \%$ de los pacientes y el $31,8 \%$ fue calificado como en riesgo de padecerlo. El trastorno depresivo estuvo presente en el $8,7 \%$ de los pacientes, y el $14,5 \%$ fue clasificado como en situación de riesgo de padecerlo. Se observó mayor prevalencia de ansiedad en el sexo femenino con 19,6\% en comparación de $12,5 \%$ en los varones. La frecuencia de diagnóstico de depresión también fue mayor en el sexo femenino (Figura 1).

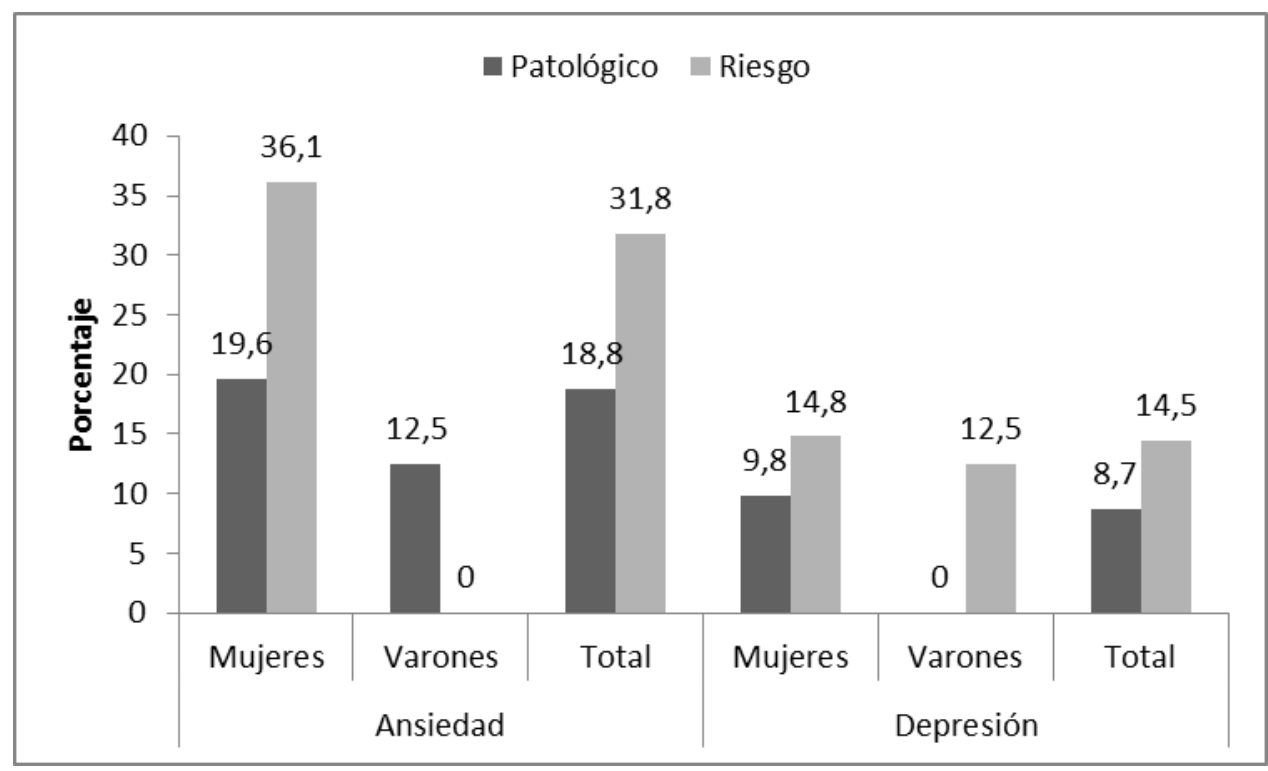

Figura 1. Trastorno depresivo y de ansiedad por la escala HAD en pacientes que consultan en la Unidad de Obesidad del Hospital de Clínicas. $n=69$ 
En cuanto a la faja etaria, el grupo con mayor prevalencia de trastornos de ansiedad fue el de mayores de 60 años (33,3\%), seguido por el grupo de 30 a 39 años $(27,7 \%)$. Para el trastorno depresivo, los menores de 30 años fueron los más afectados (18,2\%) (Tabla 4).

Tabla 4. Ansiedad y depresión por edad en pacientes que consultan en la Unidad de Obesidad del Hospital de Clínicas. $\mathrm{n}=69$

\begin{tabular}{llllll}
\hline $\begin{array}{c}\text { Faja } \\
\text { Etaria }\end{array}$ & Total & Ansiedad & \multicolumn{2}{c}{ Depresión } \\
& Riesgo & Positivo & Riesgo & Positivo \\
\hline$<30$ & 11 & $3(27,3)$ & $2(18,1)$ & - & $2(18,2)$ \\
30 a 39 & 18 & $3(16,6)$ & $5(27,7)$ & $5(27,8)$ & $2(11,1)$ \\
40 a 49 & 20 & $11(55)$ & $1(5)$ & $2(10)$ & $2(10)$ \\
50 a 59 & 14 & $3(21,4)$ & $3(21,4)$ & - & - \\
$>60$ & 6 & $2(33,3)$ & $2(33,3)$ & $3(50)$ & - \\
\hline
\end{tabular}

*entre paréntesis se observan los porcentajes

\section{Test de Imagen Corporal}

El 56,5\% de los pacientes presentó alteraciones de la imagen corporal en distintos grados (44,9\% en grado 1 y $11,6 \%$ en grado 2$)$. En la distribución por sexo, las mujeres presentaron una mayor frecuencia tanto del grado I como del grado 2 de los trastornos de la imagen corporal que los varones (Figura 2).

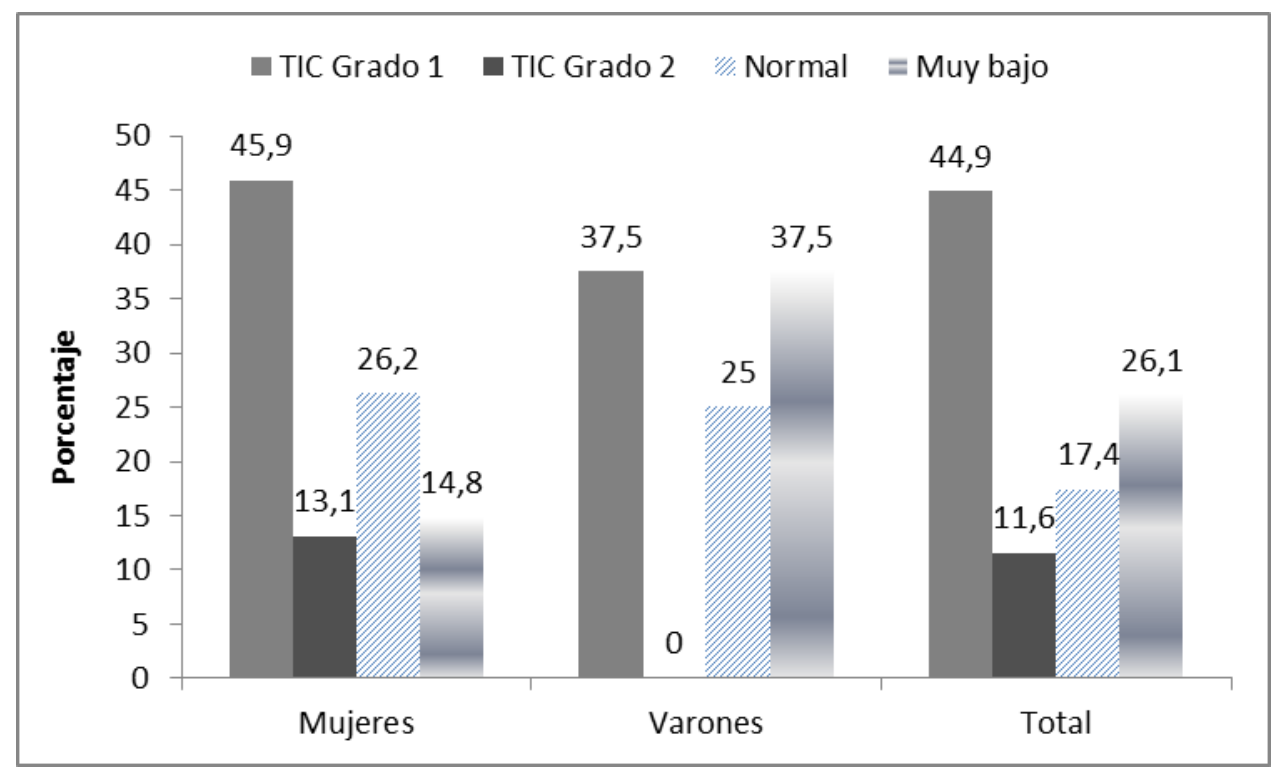

Figura 2. Trastorno de la imagen corporal distribuido por sexo en pacientes que consultan en la Unidad de Obesidad del Hospital de Clínicas. $n=69$ 
En cuanto a la distribución etaria, el grupo de 30 a 39 años fue el presentó mayor frecuencia $(27,8 \%)$ de la imagen corporal grado II (Tabla 5 ).

Tabla 5. Distorsión de la Imagen Corporal y su distribución por grupo etario en pacientes que consultan en la Unidad de Obesidad del Hospital de Clínicas. $n=69$

\begin{tabular}{lllll}
\hline \multirow{2}{*}{$\begin{array}{c}\text { Franja } \\
\text { etaria }\end{array}$} & \multicolumn{4}{c}{ Distorsión de la Imagen Corporal } \\
\cline { 2 - 5 } & Muy bajo & Normal & Grado I & Grado II \\
\hline$<30$ & $1(9,1)$ & $2(18,2)$ & $6(54,5)$ & $2(18,2)$ \\
30 a 39 & $4(22,2)$ & $2(11,1)$ & $7(38,9)$ & $\mathbf{5 ( 2 7 , 8 )}$ \\
40 a 49 & $2(10,0)$ & $8(40,0)$ & $9(45,0)$ & $1(5)$ \\
50 a 59 & $4(28,6)$ & $5(35,7)$ & $5(35,7)$ & - \\
$>60$ & $1(16,7)$ & $1(16,7)$ & $4(66,7)$ & - \\
\hline
\end{tabular}

*entre paréntesis se observan los porcentajes

Al analizar la relación de los trastornos de la imagen corporal con ansiedad y depresión, se observa que en la medida que las puntuaciones del test aumentaron, hasta llegar al trastorno de la imagen corporal grado II, también aumentó la prevalencia de ansiedad y depresión (Tabla 6).

Tabla 6. Trastornos de la imagen corporal y su relación con ansiedad y depresión en pacientes que consultan en la Unidad de Obesidad del Hospital de Clínicas. n=69

\begin{tabular}{llllll}
\hline $\begin{array}{l}\text { Imagen } \\
\text { Corporal }\end{array}$ & $\begin{array}{c}\text { Ansiedad } \\
\text { Riesgo }\end{array}$ & Positivo & $\begin{array}{c}\text { Represión } \\
\text { Riesgo }\end{array}$ & Positivo & Total \\
\hline Muy Bajo & - & $1(8,3)$ & $1(8,3)$ & - & 12 \\
Normal & $3(16,6)$ & $2(11,1)$ & $1(5,5)$ & - & 18 \\
TIC I & $17(54,8)$ & $5(16,1)$ & $4(12,9)$ & $3(9,7)$ & 31 \\
TIC II & $2(25)$ & $5(62,5)$ & $3(37,5)$ & $3(37,5)$ & 8
\end{tabular}

TIC II: Trastorno de la imagen corporal grado II

*entre paréntesis se observan los porcentajes

Respecto a los síntomas de descontrol alimentario, 52 pacientes $(75,4 \%)$ refirieron comer en respuesta a factores emocionales, $50(72,5 \%)$ comen más rápido que lo normal, 49 (71\%) experimentan culpa luego de los episodios de descontrol alimentario, $45(65,2 \%)$ comen a pesar de no sentir hambre, $30(43,5 \%)$ comen mucho, $14(20,3 \%)$ lo hacen hasta sentir náuseas. La sintomatología de descontrol con la obesidad se observó con mayor frecuencia de respuestas positivas para los grupos de mayor IMC. Al relacionarlo con los resultados de los instrumentos de medición, no se observó una mayor prevalencia en los grupos de pacientes con trastorno de ansiedad ni con pacientes en riesgo de padecerlos. Lo mismo se aplica a los pacientes con trastorno depresivo o riesgo de padecerlo, observándose incluso una mayor frecuencia en los grupos que puntuaron negativo para el diagnóstico. Si se observó mayor frecuencia de síntomas de descontrol alimentario en aquellos pacientes que presentaron trastornos de la imagen corporal, especialmente el grupo de trastornos de la imagen corporal I (Tabla 7). 
Tabla 7. Descontrol alimentario y su relación con obesidad, ansiedad, depresión e imagen corporal en pacientes de la Unidad de Obesidad del Hospital de Clínicas. $n=69$

\begin{tabular}{|c|c|c|c|c|c|c|}
\hline & & $\begin{array}{l}\text { Come } \\
\text { rápido } \\
(n=50)\end{array}$ & $\begin{array}{l}\text { Siente } \\
\text { culpa } \\
(n=49)\end{array}$ & $\begin{array}{l}\text { Come sin } \\
\text { hambre } \\
(n=45)\end{array}$ & $\begin{array}{l}\text { Come } \\
\text { mucho } \\
(n=30)\end{array}$ & $\begin{array}{l}\text { Come hasta } \\
\text { tener } \\
\text { nauseas } \\
(n=14)\end{array}$ \\
\hline \multicolumn{7}{|l|}{ IMC } \\
\hline Sobrepeso & 3 & $3(100)$ & $3(100)$ & $1(33,3)$ & $1(33,3)$ & $1(33,3)$ \\
\hline Obesidad 1 & 10 & $7(70)$ & $9(90)$ & $6(60)$ & $3(30)$ & $0(0)$ \\
\hline Obesidad 2 & 23 & $16(69,6)$ & $14(60,9)$ & $14(60,9)$ & $8(34,8)$ & $5(21,7)$ \\
\hline Obesidad 3 & 33 & $24(48)$ & $23(46,9)$ & $24(53,3)$ & $18(60)$ & $8(24,2)$ \\
\hline \multicolumn{7}{|l|}{ Ansiedad } \\
\hline Negativo & 34 & $23(67,6)$ & $24(70,6)$ & $20(58,8)$ & $12(35,3)$ & $7(20,6)$ \\
\hline Riesgo & 22 & $17(77,3)$ & $16(72,7)$ & $16(72,7)$ & $12(54,5)$ & $3(13,6)$ \\
\hline Positivo & 13 & $10(76,9)$ & $9(69,2)$ & $9(69,2)$ & $6(46,2)$ & $4(30,8)$ \\
\hline \multicolumn{7}{|l|}{ Depresión } \\
\hline Negativo & 53 & $39(73,6)$ & $37(69,8)$ & $33(62,3)$ & $21(39,6)$ & $8(15,1)$ \\
\hline Riesgo & 10 & $7(70)$ & $7(70)$ & $8(80)$ & $7(70)$ & $4(40)$ \\
\hline Positivo & 6 & $4(66,7)$ & $5(83,3)$ & $4(66,7)$ & $2(33,3)$ & $2(33,3)$ \\
\hline \multicolumn{7}{|c|}{ Imagen corporal } \\
\hline Normal & 18 & $16(88,9)$ & $12(66,7)$ & $13(72,2)$ & $8(44,4)$ & $1(5,6)$ \\
\hline Muy Bajo & 12 & $6(50)$ & $7(58,3)$ & $4(33,3)$ & $3(25)$ & $2(16,7)$ \\
\hline TICI & 31 & $21(67,7)$ & $22(71)$ & $22(71)$ & $14(45,2)$ & $7(22,6)$ \\
\hline TICII & 8 & $7(87,5)$ & $8(100)$ & $6(75)$ & $5(62,5)$ & $4(50)$ \\
\hline
\end{tabular}

*entre paréntesis se observan los porcentajes

\section{DISCUSIÓN}

La población que acude en busca de tratamiento integral para la obesidad en nuestro país, mostró ser una población predominantemente femenina, en la edad media de la vida y con educación terciaria. El sexo masculino está representado por menores de 30 años. El $76,3 \%$ de los pacientes tenía un IMC mayor a $35 \mathrm{~kg} / \mathrm{m}^{2}$.

Más de la mitad de los pacientes en el estudio, tenía un tiempo de obesidad mayor a 10 años a la fecha de la primera consulta y $17,5 \%$ no había intentado bajar de peso antes o solo lo había intentado en una oportunidad a pesar de que como ya se mencionó, más de $75 \%$ de los pacientes tenía una obesidad de grado II o III. Estos hallazgos son llamativos y podrían tener relación con la percepción del propio estado en la población de obesos. Truesdale y Stevens en un estudio en población general encontraron que el $72 \%$ de las mujeres obesas y el $73,3 \%$ de los hombres obesos se consideraban a sí mismos con sobrepeso y el $5,6 \%$ de las mujeres y $20 \%$ de los hombres, dentro del peso normal (15). Joneso et al. hablan de hasta $50 \%$ de los obesos clasificándose a sí mismos como en rango de sobrepeso (16). Aparentemente un alto porcentaje de la población obesa no se identifica con este diagnóstico, experimentan menor angustia frente a la pérdida de control alimentario y a la sobrealimentación. Esta falta de introspección respecto al diagnóstico nutricional podría explicar el alto porcentaje de pacientes con obesidad de larga data y pocos intentos de cambiar la situación metabólica en la que se encuentran.

Los hábitos alimentarios de los pacientes fueron analizados y el $75 \%$ de los pacientes refirió comer en respuesta a factores emocionales (cuando se sentían tristes, nerviosos o enojados). El "comer emocional" se refiere a la tendencia a comer en respuesta a 
emociones negativas (5). Diversas teorías intentan explicar el comer emocional como una estrategia de reducción de la conciencia del distres a través de un bloqueo o disociación (17). Otras hablan de su relación con la Alexitimia, factor que ha mostrado su asociación a los Trastornos Alimentarios, así como al comer emocional $(5,6,8)$. La Alexitimia está definida como la incapacidad para identificar y expresar emociones, así como un pensamiento orientado hacia el exterior (18). Agnieszka et al. reportaron una prevalencia de $46 \%$ de alexitimia en pacientes obesas (8), mientras que Pinaquy et al. reportaron una frecuencia similar, $52 \%$ de los pacientes obesos fueron calificados como alexitímicos (6). Los hallazgos de estudios sobre Alexitimia en obesos señalan que aquellos que presentan esta dificultad para identificar y comunicar sus emociones también presentan tendencia marcada a comer en respuesta a emociones. Los déficits afectivos y el comer emocional están ligados $(5,6,8)$. Sería de gran valor la exploración de Alexitimia en nuestra población de pacientes obesos, ya que serviría para diseñar estrategias de manejo de síntomas de alta frecuencia en la población, como el comer emocional.

Se recogieron también otros hábitos alimentarios: comer mucho, comer rápido, comer hasta sentirse incómodo o con náuseas, comer aun sin apetito y la culpa que generan los episodios de falta de control al comer, hábitos que se encuentran dentro de los síntomas de Trastorno por Atracones (criterio B del DSM V) (3). Estos síntomas se observaron en la población en frecuencias bastante elevadas, entre $20 \%$ (correspondiente a comer hasta sentirse con náuseas) y $75 \%$ (correspondiente a come mucho). La máxima frecuencia se dio en el grupo de pacientes con sobrepeso, tal vez por estar constituido este grupo por muy pocos pacientes (solo 3) y luego con frecuencia ascendente paralela al grado de obesidad. En cuanto a la asociación de síntomas de Trastorno por Atracones con Ansiedad - Depresión no se observó una mayor frecuencia en aquellos con puntuaciones más altas en las escalas HAD A y HAD D. Si bien no fue uno de los objetivos de este estudio determinar la prevalencia de Trastorno por Atracones, si se tuvo acceso a síntomas que construyen el diagnóstico. En este sentido, se esperaría que los pacientes con estos síntomas presentaran mayor frecuencia de psicopatología asociada, ya que la literatura internacional reporta que los pacientes obesos con Trastorno por Atracón presentan más síntomas de Depresión y Ansiedad que los pacientes obesos que no reúnen criterios diagnósticos de Trastorno por Atracón, e incluso propone que el Trastorno por Atracón podría mediar la relación entre obesidad y Depresión $(5,19,20)$.

La prevalencia de Trastornos de Ansiedad en la muestra fue de 18,8\% medida con la escala HAD A. Estos datos están dentro de las tasas encontradas por literatura previa, que habla de prevalencias que varían entre $17,7 \%$ y $38,8 \%(8,10)$, e incluso un estudio multicéntrico presentó tasas bastante más bajas, entre $4 \%$ y $7,5 \%$ para países de América Latina y el Caribe (11). El $32 \%$ de los pacientes se presentó como una población "en riesgo", lo que significa que presentaron síntomas de tipo ansiosos pero de forma leve. La distribución por sexos fue mayor para el sexo femenino y el principal grupo etario afectado fue el conformado por pacientes mayores de 60 años.

La prevalencia de Depresión fue $8,7 \%$ medida con la escala HAD D. Prevalencias algo mayores fueron reportadas por otros estudios, como el de Agnieszka et al. que reportó una prevalencia de $12 \%$ de depresión en pacientes buscando tratamiento por obesidad. Otros estudios hablan de prevalencias similares a las observadas en el presente estudio, 4 a $10 \%$ en poblaciones latinas, y $7,2 \%$ en población asiática $(8,10,11)$. Hasta $14,5 \%$ de los pacientes de la muestra se presentó como población en riesgo de padecer Depresión. La Depresión al igual que la Ansiedad, fue más frecuente en las mujeres y en aquellos pacientes menores de 30 años.

Varios estudios documentan la relación entre la obesidad y la depresión. Metaanálisis han encontrado una fuerte asociación tanto de obesidad con depresión como de depresión con obesidad (21), y los factores que se han propuesto como mediadores han sido la alexitimia y el comer emocional (5). Goldschmit et al. en su estudio sobre afectividad en obesos, encontraron que el comer emocional media la relación entre la obesidad y el IMC, de manera que el comer emocional podría desencadenar o mantener la obesidad y esto llevar al empeoramiento de los síntomas depresivos (6). También se ha descrito una mayor frecuencia de Ansiedad y Depresión en pacientes que presentan diagnóstico de Trastorno por Atracones (5-8). Como ya se mencionó previamente, en el presente estudio se han encontrado prevalencias elevadas de comer emocional, así como de síntomas de Trastorno por Atracones, sin embargo las diferencias en frecuencias son solo levemente mayores y no resultaron estadísticamente significativas en los análisis. Esto podría 
deberse a que estos síntomas no fueron recogidos con instrumentos validados para tal fin, si no que se accedió a ellos a través de las fichas clínicas, que fueron llenadas por profesionales de áreas distintas a la salud mental y con autorreporte de los pacientes.

En cuanto a la imagen corporal, $56,5 \%$ de los pacientes presento algún grado de Trastorno respecto a su Imagen Corporal, siendo más frecuentes en los pacientes menores de 40 años y en las mujeres. La principal molestia respecto a la imagen o silueta reportada por los pacientes fue el tamaño corporal a la hora de elegir ropa y la culpa respecto a su problema de peso. La literatura disponible respecto al grado de satisfacción con la imagen corporal se basa en estudios realizados en población adolescente, enfocándose apenas en este aspecto de la población adulta. Sin embargo en estudios en adolescentes, la insatisfacción con la imagen corporal está asociada a baja autoestima y a depresión (22).

La inclusión de la dimensión cognitivo emocional en el tratamiento de la obesidad en nuestro medio es incipiente, y por tal motivo resulta de vital importancia sentar las bases para desarrollar intervenciones efectivas, tanto terapéuticas como de prevención. Si bien el presente estudio arroja datos concluyentes al respecto, resultaría de importancia la aplicación de otros instrumentos validados para realizar un diagnóstico certero de otras entidades psicopatológicas, como el Trastorno por Atracones, que pudieran requerir un enfoque de tratamiento específico.

\section{REFERENCIAS BIBLIOGRAFICAS}

1. WHO [homepage on internet]. Centro de Prensa. Nota descriptiva $N^{\circ}$ 311. $2014-$ [cited on 2014 novembre 4]. Available from:

http://www.who.int/mediacentre/factsheets /fs311/es/

2. World Health Organization. "CIE 10: Trastornos mentales y del comportamiento: descripciones clínicas y pautas para el diagnóstico." (1992).

3. American Psychiatric Association. Diagnostic and statistical manual of mental disorders, 5th edn. American Psychiatric Publishing 2013, Arlington

4. Cordella P. ¿̇ncluir la obesidad en el Manual de Enfermedades Mentales (DSM-IV)? Revista chilena de nutrición. 2008 Sep;35(3):181-7.

5. Pinaquy S, Chabrol H, Simon C, Louvet JP, Barbe P. Emotional Eating, Alexithymia, and Binge-Eating Disorder in Obese Women. Obesity Research. 2003;11(2):195-201.

6. Goldschmidt $A B$, Crosby RD, Engel SG, Crow SJ, Cao L, Peterson CB, Durkin N. Affect and eating behavior in obese adults with and without elevated depression symptoms. International Journal of Eating Disorders. 2014;47(3):281-6.

7. Faith MS, Butryn M, Wadden TA, Fabricatore A, Nguyen AM, Heymsfield SB. Evidence for prospective associations among depression and obesity in population-based studies. Obesity Reviews. 2011;12(5):e438-53.

8. Źak-Gołąb A, Tomalski R, Bak-Sosnowska M, Holecki M, Kocełak P, OlszaneckaGlinianowicz $M$ et al. Alexithymia, depression, anxiety and binge eating in obese women. The European Journal of Psychiatry. 2013 Sep;27(3):149-59.

9. Solmi F, Hatch S, Hotopf M, Treasure J, Micali N. Prevalence and correlates of disordered eating in a general population sample: the South East London Community Health (SELCOH) study. Soc Psychiatry Psychiatr Epidemiol 2014; 49:1335-46.

10. Lin HY, Huang CK, Tai CM, Lin HY, Kao YH, Tsai CC et al. Psychiatric disorders of patients seeking obesity treatment. BMC psychiatry. 2013;13(1):1.

11. Scott KM, Bruffaerts R, Simon GE, Alonso J, Angermeyer M, de Girolamo G et al. Obesity and mental disorders in the general population: results from the world mental health surveys. Int $\mathrm{J}$ Obes (Lond) 2008;32:192-200.

12. Bjelland I, Dahl A, Haug T, Neckelmann D. The validity of the Hospital Anxiety and Depression Scale. An updated literature review. Journal of Psychosomatic Research 2002;52:69-77.

13. Behar R. La construccion cultural del cuerpo: El paradigma de los trastornos de la conducta alimentaria. Rev Chil NeuroPsiquiat 2010;48(4):319-34.

14. Bucchianeri MM, Arikian AJ, Hannan PJ, Eisenberg ME, Neumark-Sztainer D. Body dissatisfaction from adolescence to young adulthood: Findings from a 10-year longitudinal study. Body image. 2013;10(1):1-7.

15. Truesdale K, Stevens J. Do the obese know they are obese? N C Med J. 2008; 69(3):188-94.

16. Jones M, Grilo C, Masheb $R$, White $M$. Psychological and Behavioral Correlates of Excess Weight: Misperception of Obese Status among Persons with Class II Obesity. Int J Eat Disord. 2010;43(7):628-32.

17. Ouwens MA, van Strien $T$, van Leeuwe JF. "Possible pathways between depression, emotional and external eating. A structural 
equation model." Appetite 2009;53(2):2458. doi:10.1016/j.appet.2009.06.001.

18. Carneiro B, Pinzao E. Alexitimia: Uma revisao do Conceito. Psicologia: Teoria e Pesquisa Jan-Mar 200;25(1):103-8.

19. De Zwaan M. "Binge eating disorder and obesity." International journal of obesity and related metabolic disorders: journal of the International Association for the Study of Obesity 2001;25,Suppl 1, S51-5.

20. Peterson R, Latendresse S, Bartholome L, Warren C, Raymond N. Binge Eating Disorder Mediates Links between Symptoms of Depression, Anxiety, and Caloric Intake in Overweight and Obese Women. Journal of Obesity. 2012; 2-8. studies. Archives of general psychiatry. 2010;67(3), 220-9

22. Paxton SJ, Neumark-Sztainer D, Hannan PJ, Eisenberg ME. Body dissatisfaction prospectively predicts depressive mood and low self-esteem in adolescent girls and boys. Journal of clinical child and adolescent psychology. 2006;35(4):539-49.

23. González Vera, M. Patriarcado, machismo e impunidad: causas de la cotidiana violencia de género En: Coordinadora de Derechos Humanos del Paraguay (Codehupy). Derechos humanos en Paraguay/ Yvypóra Derécho Paraguáipe. Codehupy. Asunción: Mercurio S.A.; 2012. pp. 447-63.

21. Luppino FS, de Wit LM, Bouvy PF, Stijnen T, Cuijpers $P$, Penninx BW. Overweight, obesity, and depression: a systematic review and meta-analysis of Iongitudinal 